\title{
VISUAL PREFERENCES FOR EDUCATIONAL GAME DESIGNS THROUGH THE GRAPHIC STYLE APPROACHES
}

\author{
Lilian Lee Shiau Gee ${ }^{1}$ \\ Jasni Dolah ${ }^{2}$ \\ Victor B. Pangayan ${ }^{3}$ \\ Fakulti Kemanusiaan, Seni dan Warisan, Universiti Malaysia Sabah, Kota Kinabalu, Sabah ${ }^{1 \& 2}$ \\ Pusat Pengajian Seni, Universiti Sains Malaysia, Pulau Pinang, Sabah ${ }^{3}$ \\ lilian@ums.edu.my,jasnidolah@usm.my,victorpangayan@ums.edu.my \\ Tarikh diterima: 25 Julai 2019 / Tarikh dihantar: 9 September 2019
}

\begin{abstract}
The role of visual design in educational games is not only for beauty, but also for critical motivation to engage students in nurturing emotional values and thus enhancing their positive experiences. In terms of visual design, graphic style is key to re-evaluating the power and suitability of visual design in variety and rapidly changing game. This paper discusses the importance of graphic styles and their impact on educational games in the context of improving students' learning through play. The influence of design based graphic styles, the graphic characterization and the relationship of graphic trend in design innovation should be emphasized to determine how design can influence the game visualization.
\end{abstract}

Keywords: Visual design, graphic styles, educational game, game art, experiences.

\section{INTRODUCTION}

The success of a digital game is closely related to the strength of the game designer to produce a game that matches the player preferences. Visual game is realized as an important aspect of game design in order to heighten the player's experiences (Lin, Lin \& Yang, 2015; Solarski, 2013; McLaughlin, Smith \& Brown, 2010). The visual design makes the basis game development as to infuse a positive experience through emotional involvements of the players in the game. Consequently, the resulting optimal experience is based visualization presented in the game. In depth, 
the visual design aspect of the game is artistically called 'graphic style,' where certain graphic styles bring a different visual appeal to the player. Understanding of the graphic style applied in the game as a practical approach to accommodate what players want in the game as well as the overall presentation of the game with specific objectives and target players such as educational games.

\section{Designing Challenges for Educational Games}

The evolution of games in the marketplace is shifting apace, from genre to graphic style, bringing a major focal point to educational games. The use of games in education led by technology brings new changes in learning and teaching in today's classroom (Osman \& Bakar, 2012). Advantages and encouragement of the use of educational games in school settings bring about strong efforts to research the importance and effectiveness of educational games in Malaysia, including educational game development and innovation, as well as strategic and educational game operations following the school education curriculum.

Nonetheless, in Malaysia, the implementation of educational games is still in early stages (Roslina \& Azizah, 2009; Rozana \& Roslina, 2018) and visual design is a phenomenon that is less emphasized in educational games research (Deen, 2015). The lack of attractive design (Whittion, 2011; Soylucicek, 2012; Dickey; 2015) will be a major obstruction to the utilization of educational games among students. Hiebert (2009) and David and Glore (2010) found that educational games less pressing consideration to the importance of visual design in the game, which influence game appearance and student interest in overall game visualization. The results of the pilot study by Lilian and Jasni (2016) shows that visuals are a major factor for students' concern in the context of continuous play. Visuals are the first component that students perceive to attract and engage in deeper engagement which in turn motivates activity to play in the learning and teaching process. This indicates that the visual design as a player's attraction and emotion as well as an indication of the strength of the educational game intended for local students, which creates a space for discussion, is to realize the visual concept applied as a specific representation (variable) which able to provide a good response between the player and the visually context of the game.

Concentrating on the visual design possibilities, which is something that is always questionable and should be identified by the graphic style used (Lin et al., 2015; Paterson, 2007; Mohr \& Gleicher, 2001). Certain graphic styles will give the person a different visual appeal based on the person's experience and taste, thus answering a different responses and interests. However, the suitability of a 
particular graphic style in the game with focus groups still lack empirical data and are not generally available (Demers, 2002). With that, the graphic style is a prolongation of work that needs to be practiced and focused on producing high art direction in terms of visual design for the educational game developments to meet the local student needs. This has led to visual design considerations that need to be expanded in terms of graphic style to outline the possibility that they create an overall atmosphere and decoration to create a positive student experience.

\section{Sparked of 'Graphic Styles'}

Generally, the graphic style is an overall work of art that shapes the whole visualization to create the atmosphere and game environment (Meigs, 2003; Lin et al., 2015). The graphic style is also the dominance of actions that represent different visual entities in game design (Egentfeldt-Neilsen et al., 2015), where the characterization of the graphic style is influenced by various mainstream and traditional art-visual arts genres (Mohr \& Gleicher, 2001). As Adams (2014) points out, the style of graphics applied is in line with the design principles for designing the game outlook. This indicates how the art direction practice and the art characterization of the graphic style is to identify the visual design approach aimed at the game.

With the advancement and importance of the game's appearance in recent years it has created varying levels of appearance with an emphasis on graphic style in various (low and high) details. With the evolution of video game design approach has sparked a debate on a better appearance of 'Abstract' and 'Realistic' style (Gulick, Burnett \& Coulton, 2017). Some digital games may be more fitted to a particular graphic style and differences in graphics style bring knowledge to the suitability of a certain style that can evoke player behaviour and reaction while playing. This also reviewed at educational games, which graphic style should suit the target students. The strong argument between 'Abstract' and 'Realistic' styles (Lindh, 2018; Bollini, 2017) has been referred to in educational games as the nature and medium of communicating information and learning processes visually. Because of this, there are disputes between the possibilities between 'Abstract' and 'Realistic' styles that can be offered to students.

From the 'Abstract' point of view, abstraction is an ideas that facilitates the information transmissions that based on McCloud's theory of "amplification through simplification" which is more effective to be understood and accepted by students. The increasing popularity of video games in the marketplace boasts of 'Abstract' style (Egenfeldt-Nielsen et al., 2015) which holds to the principle of 
the importance of simplicity in gaming as a factor contributing to the observation of 'Abstrac' images in educational games.

Gullick et al. (2017) emphasizes that most game design studies less evaluate and considering the 'Abstract' style towards the players. In the view of Perry (2009) and Smeureanu and Isăilă (2017), the importance of the 'Realistic' style is relevant to the tendency of the relationship of imagination, especially the actual experience that educational games bring to the classroom. Realistic characters and environments are a stimulus for ideas in video games to generate strong imagination among players (Schroth, LaValle, Iype \& Sheppard, 2015). Realistic looks became a major trend in the 1990's, however, and there is still a lack of data and research on the importance of visual realism in educational games in terms of student acceptance, especially their emotional immersion and experiences (Selmbacherova, Sisler \& Brom, 2014).

In this situation, the appropriateness of the graphic style applied is crucial to being able to draw the students' attention and perceptions into the visual concepts presented in the game as to assist students learning process through gameplay activities. Critical views of the 'Abstract' and 'Realistic' styles have a strong basis for ongoing discussion with visual design paradigms that need to be adapted to the game screen. The focal point is on how graphic style affects the interaction between students and educational games and the potential of graphic styles to support educational games. Graphic style application strategies in educational games are important to enhance the student experiences. Therefore, understanding and integrating elements of artwork that complement each other in the 'Abstract' and 'Realistic' styles are also part of the visual design, namely, to deepen the interpretation of visual presentation in the game (Lindh, 2018; Bollini, 2017).

\section{The 'Abstract' and 'Realistic' Styles}

The word 'style' is frequently connected with the method of performing art through the placement of specific content features based on elements of art, technique, expression and inspiration as determined by the artist's choice. The divergences in the graphic style of presentation can be viewed in terms of the artistic quality associated with the elements of artistic creation in detail (Gulz \& Haake, 2006) which can be understood in the artwork. Similar to video games, graphic styles are also classified based on different components. The variety of graphic styles applied is the art concept that designers want to convey in the characters, game assets, objects and environments. 
McLaughlin et al. (2010) and Egenfeldt-Nielsen et al. (2015) classify specific graphics styles for video games. The first style mentioned is the 'Abstract' style. The concept of abstraction is a visual representation that has no direct bearing on the real world (Lauer \& Pentak, 2012). This style is more akin to a minimalist concept by conceptualizing the actual image by simplifying details, especially the shape and form. As such, the 'Abstract' style prefers to flattening, simplify and rearrange the form along with the utilization of low fidelity and nondetailed art elements to produce a visual approximation of the game. Usually the 'Abstract' style in the game is related to the use of symmetrical lines, geometry, flat colour and shading to express a particular feeling and meaning to the players. Visualisations of the character and environment in the simple form are categorized into abstraction. As for the 'Realistic' style, it is more like representing the real world with a high degree of fidelity. In general, the work to enrich realistic perceptions is focused on high detail in terms of shape, colour, line and lighting. In virtual games, this style is often characterized by a high degree of processing in depicting the scene (characters, environments and assets) with a tendency to look natural.

\section{Implementation of 'Abstract' and 'Realistic' Style}

Just like formal artwork, the concept of classifying graphic styles is based on elements of art in traditional art with specific design ratios to produce a unique representation mode to visualize the games. In this context, three key elements have been outlined by McLaughlin et al. (2010) and Egenfeldt-Nielsen et al. (2015) to classify 'Abstract' and 'Realistic' styles mainly the shape, texture and shading. In this study, the 'Abstract' style is based on a minimalist design approach, which is designed in two-dimensional form with simple shapes, solid colours, clear typography, limited textures and movement.

Abstract design avoids drop shadows, bevels, embosses and gradients. In terms of form more focused on simple and geometric shapes, then the shades are toon shading and textures are limited to painted texture. As such, the 'Abstract' style is intensified with these features to create a visually appealing appearance. While the 'Realistic' style emphasizes the role of artificial textures, drop shadows, emboss and gradients appear for the intent of presenting a detailed visual appearance. In this study, the realistic style is based on the use of three polygon-shaped densities, the mapping technique based on '3D modelling' and the proportional proportions that tend to 'smooth blended appearance' or 'smooth shading' realistic. For example, Figure 1 shows visual representations of characters that are 'Abstract' and 'Realistic.' In this study, realistic style is more 
oriented to the use of three-dimensional in polygons shaped, mapping texture technique on the object surface, blended stroke proportions or known 'smooth shading' that tend to look more naturalistic. For example, Figure 1 shows a conceptual overview of characteristic of 'Abstract' and 'Realistic' styles.

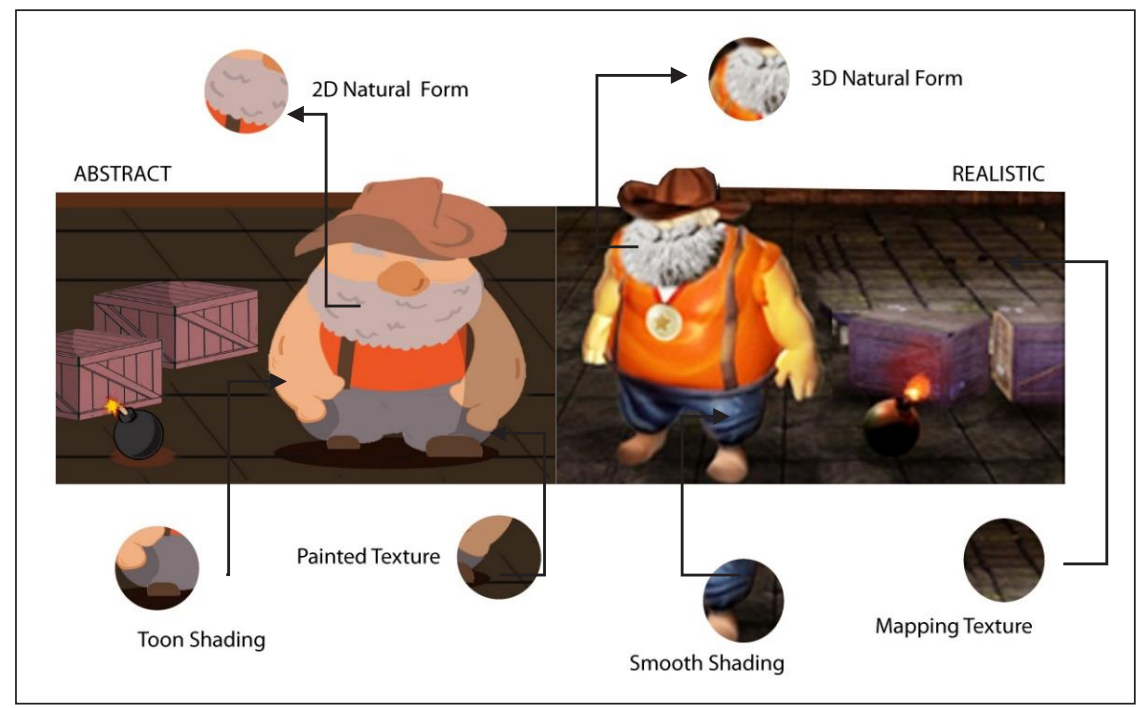

Figure 1 The overview characteristics of 'Abstract' and 'Realistic' styles

Consequently, the underlying rules of graphic style (abstract and realistic) are grounded in the constituents and rules of artistry as the art characteristics to create a specific and consistent art directed towards the educational games.

\section{Relevance of Factors by Forms, Textures and Shadings}

The effectiveness of visualization presented to students is based on a proper visual design and this depends on the graphic style that is interpreted and represents the game appearance. Each graphic style has different artistic properties, and these features are also elements in creating and displaying the style. Thus, seeing the complementary and combining elements of artwork is likewise part of the visual design, which is to deepen the graphic style practice as a visual representation of the game.

The consequences of student feedback indicate that as a visual conceptualization the educational game should be outfitted with a 'natural form' in the 'Abstract' and 'Realistic' style to provoke student interests. The 'natural form' is the catalyst that enhances involvement and stimulates visual satisfaction in the educational games that incorporate with abstraction and realistic representation 
mode. In addition, Solarski (2013) states that curved and natural forms often seem friendlier and more well-liked by the public. Although the texture tends to be added, created or simulated to look and feel surplus to describe the visual appearance. However, student satisfaction mostly focuses on the natural shape and shading as impressive and pleasure element to generate stimulus appeal for each graphic style. Table 1 depicts the comparative analysis of specific elements of art that the students emphasized in the 'Abstract' and 'Realistic' styles.

Table 1 The design features for 'Abstract' and 'Realistic' style

\begin{tabular}{|c|c|c|}
\hline Compare Design Features & Abstract & Realistic \\
\hline \multicolumn{3}{|l|}{ Form } \\
\hline - Natural Form & & \\
\hline - Geometric Form & - & - \\
\hline \multicolumn{3}{|l|}{ Texture } \\
\hline - Painted texture & - & - \\
\hline - Photo Texture & - & - \\
\hline \multicolumn{3}{|l|}{ Shading } \\
\hline - Smooth Shading & - & 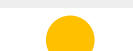 \\
\hline - Toon shading & & - \\
\hline
\end{tabular}

Overall, the interview information from the students revealed that the shading added to the illustrations of natural form objects were a great combination that produced beautiful visuals, entertaining and producing impressive sentiments. The game character and the environment are depicted in a 'natural form' to create a sense of harmony and are seen as a pleasant and gentle look to highlight the visualization of educational games. Furthermore, shading interventions are a benefit for ease readability, visibility and visual pleasure in the educational games.

\section{Contextual Framework as a Design Guidelines}

Feedback and explanatory comments have deepened the idea of the 'Abstract' and 'Realistic' style concepts that have the potential to meet the needs and desires of local students. The reaction of integrating art elements in graphic style and the concept of art elements between the 'Abstract' and 'Realistic' styles, supports the robustness of the design guidelines developed for educational games. The valuation of the art elements shows that the 'Abstract' style tends to depend on the features associated with natural form and toon shading, and the 'Realistic' style tends to rely on natural form and smooth shading. The diversity and categorization 
of art elements give an insight into the importance of organizing art elements in enhancing the art direction of graphic style by providing optimum visualization and atmospheres. The design guidelines for the 'Abstract' and 'Realistic' styles are outlined in relation to the schematic representation as illustrated in Figure 2.

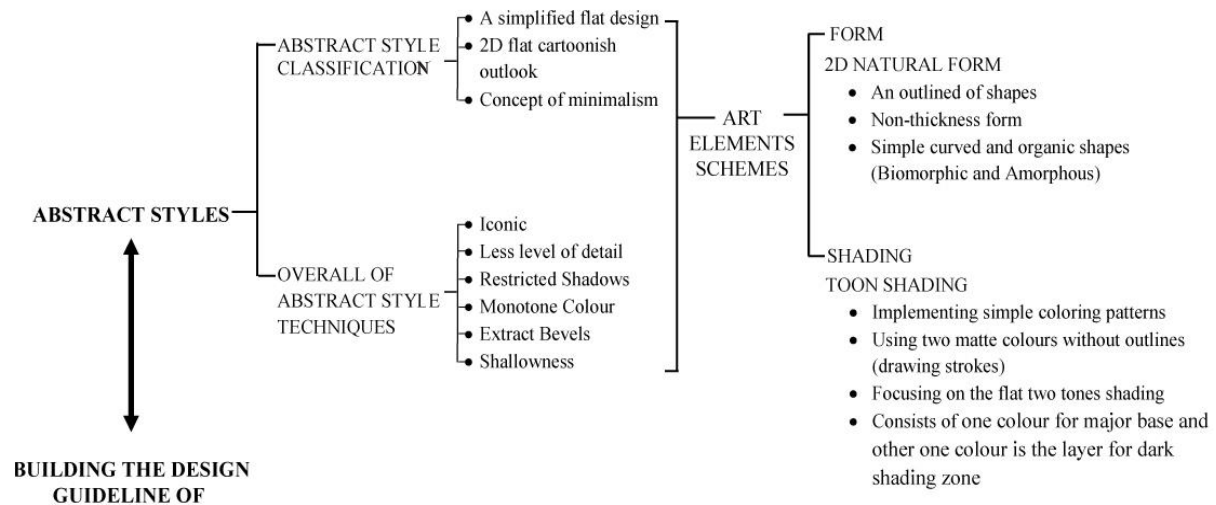

$$
\text { GRAPHIC STYLES }
$$

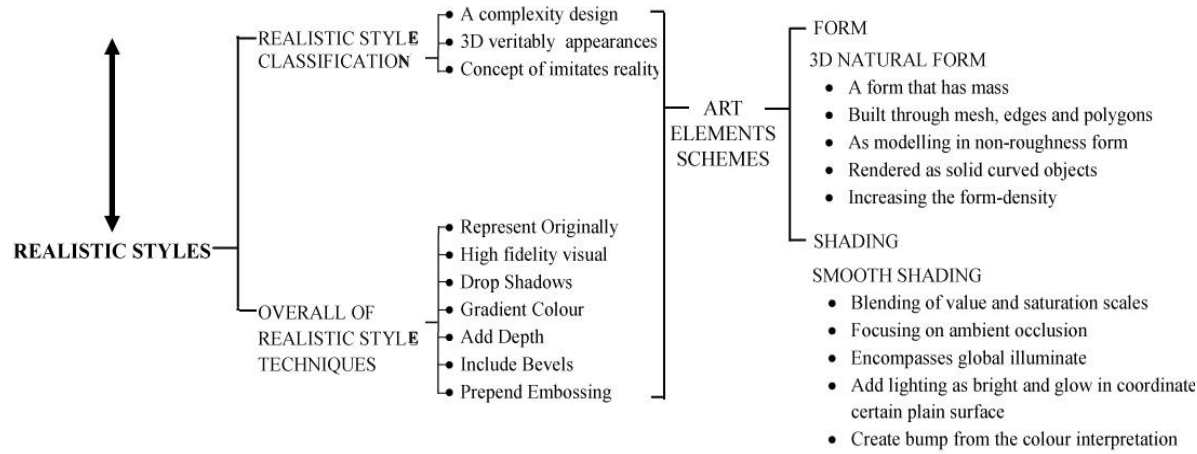

Figure 2 The principles design guideline of 'Abstract' and 'Realistic' style

The concept of visualization for each graphic style in detail - as relevant methods and techniques for incorporating graphic style in visual games. This guide acts as an input source for the placement of art elements in educational games to present unique and specific visualization features. Through the guidelines, a set of visual perspectives has been worked to realize the profundity of the graphic style in how the visual design of the game in 2D and 3D visualization was placed and directed to the students. Based on these guidelines enable full visual design innovation, including planning (action-making), design thinking (ideas and concepts), and design strategies (design processes) in educational game development. 


\section{CONCLUSION}

From a visual standpoint, game design is an artistic game with a 'game feel' to support meaningful gameplay activity. The process of designing a game as a complex visual activity to 'visualize' educational games, and requires thoughtful knowledge of graphic style to influence the student experiences. Further, students will choose educational games based on the needs, interests and suitability of the visual appearance, where visual aspects are the first thing students see (Lilian \& Jasni, 2016) before engaging in the game.

This study emphasizes the importance of visual design in educational games from graphic style planning until the integration of appropriate art elements to represent effective game play in learning activities. 'Abstract' and 'Realistic' styles include design theory, art direction, theme and combining shapes, texture and shading to create eye-catching visualizations to produce as many positive experiences as possible. This study proposes additional design values to design educational games more creatively, to cater to local students' tastes and to enhance their motivation, engagement and enjoyment.

Through the understanding of graphic style, to provide a design-led approach to enhance the innovation and competitiveness of educational games in the marketplace, and as a way of finding the suitable means to explore the field of design thinking to use creativity and empathy for the development of educational games in Malaysia. Understanding this study is important in contributing to the broad field of visual game design where visual aspects are still an important agenda in the development of a game as an ICT tool (Deen, 2015), and are considered an important factor for a successful educational game (Whitton, 2011; Dickey, 2015).

These guidelines can also be utilised as a map by designers when providing visual concepts to educational games and support to identify appropriate improvements to the situation and visualization environment that would be presented. The study also recommends a graphical style structure that is speculative as a basic reference that is expected to assist and guide experts, reduce the cost of resources and shape the expectation of student experience, thus enabling more efficient and effective representation and educational game developments. 


\section{Future Research}

In addition to recognizing the importance of visual aspects of the game, further research is recommended, such as furthering empirical research on the influence of artistic elements such as colour (Milam, 2012; Friedman, 2015) and space (Plass et al., 2009), further exploration with other types of graphic styles and further links from the 'Abstract' and 'realistic' style categories, for example, conducting research with specific categories to the 'realistic' style (enhanced realism, simplied realism and distorted realism) to significantly shape the visual pattern of educational games. Also, to identify how visual design innovations can attract interaction in specific learning subjects such as language, math and science, and the decision and implementation of the type of graphics style in the game environment that is specific to the player's characteristics, such as player age.

\section{REFERENCES}

Adams, E. (2014). Fundamentals of game design (3rd ed.). London: Pearson Education, Inc.

Bollini, L. (2017). Beautiful interfaces. From user experience to user interface design. The Design Journal, 20 (1), S89-S101.

David, A. \& Glore, P. (2010). The impact of design and aesthetics on usability, credibility, and learning in an online environment. Online Journal of Distance Learning Administration, Xiii (IV).

Deen, M. (2015). G.A.M.E Games Autonomy Motivation \& Education. Eindhoven, Netherlands: Eindhoven University of Technology Library.

Demers, O. (2002). Digital texturing and painting. San Francisco, CA: New Riders Publishing.

Dickey, M. (2015). Aesthetics and design for game-based learning. United Kingdom: Routledge, Taylor \& Francis.

Egenfeldt-Nielsen, S. (2005). Beyond edutainment: Exploring the educational potential of computer games. IT - København, Denmark: University of Copenhagen.

Egenfeldt-Nielsen, S., Heide Smith, J., \& Tosca, P. (2015). Understanding video games: The essential introduction (2nd ed.). United Kingdom: Routledge, Taylor \& Francis Group.

Friedman, A. (2015). The role of visual design in game design in games and culture. 10 (3), 291-305. Thousand Oaks, CA: Sage Publications.

Gullick, D., Burnett, D., \& Coulton, P. (2017). Visual abstraction for games on large public displays. Proceedings of 8th International Conference on Intelligent Technologies for Interactive Entertainment (pp. 271-275). Springer.

Gulz, A. \& Haake, M. (2006). Visual design of virtual pedagogical agents: Naturalism versus stylization in static appearance. 6th International Conference on Intelligent Virtual Agents, IVA, 1-9. 
Hiebert, E. (2009). High-leverage action \#3: A picture is worth a thousand words. Retrieved March, 3, 2016 from http://www.textproject.org/franklyfreddy/highleverage-action-3-a-picture-is-worth-a-thousand-words

Lauer, D., \& Pentak, S. (2012). Design basics (8th ed.). Wadsworth: Cengage Learning.

Lilian, Lee Shiau Gee \& Jasni Dolah. (2016). Graphic styles appearance in educational games to enhance Malaysian students learning: A preliminary study. Proceedings of the 2nd International Conference on Creative Media, Design \& Technology (REKA 2016).

Lin, Y., Lin, H., \& Yang, Y. (2015). Players' value structure in digital games. Games and Culture, 1-28.

Lindh, M. (2018). Beyond a skeuomorphic representation of subtractive synthesis. International Conference on Intelligent User Interfaces, IUI, 2068.

McCloud, S. (1994). Understanding comics: The invisible art. New York: Harper Collins.

McLaughlin, T., Smith, D., \& Brown, I. A. (2010). A framework for evidence based visual style development for serious games. Proceedings of the Fifth International Conference on the Foundations of Digital Games, ACM, 132-138.

Meigs, T. (2003). Ultimate game design. Building game worlds. USA: McGraw Hill.

Milam D., El-Nasr M., Bartram L., Aghabeigi, B., \& Tan, P. (2012). Similarity in visual designs: Effects on workload and performance in a railed-shooter game. Herrlich M., Malaka R., \& Masuch M. (eds.). Entertainment Computing - ICEC 2012. ICEC 2012. Lecture Notes in Computer Science, Vol. 7522. Berlin, Heidelberg: Springer.

Mohr, A., \& Gleicher, M. (2001). Non-invasive, interactive, stylized rendering. Proceedings of the 2001 Symposium on Interactive 3D Graphics, 175-178.

Osman, K. \& Bakar, N. (2012). Educational computer games for Malaysian classrooms: Issues and challenges. Asian Social Science, 8 (11), 75-84.

Paterson, I. (2007). Experiencing architectural interiors and exteriors in computer games. International Journal of Architectural Computing, 5 (1), 128-143.

Perry, F. (2009). The construction game - Using physical model making to stimulate realism in construction education. Journal for Education in the Built Environment, 4 (1), 57-74.

Plass, J., Homer, B., \& Hayward, E. (2009). Design factors for educationally effective animations and simulations. Journal of Computing in Higher Education, 21 (1), $31-61$.

Roslina, I., \& Azizah, J. (2009). Educational games (EG) design framework: Combination of game design, pedagogy and content modelling. Proceedings of the 2009 International Conference on Electrical Engineering and Informatics, ICEEI 2009, 1 (August), 293-298.

Rozana, I., \& Roslina, I. (2018). Implementation of a participatory design process in designing an educational mobile game for primary school students: A case study in Malaysia. The Adjunct Proceedings of ACM TVX2018, June, 2018, Korea.

Schroth, O., LaValle, A., Iype, D., \& Sheppard, S. (2015). Serious games as a tool for the landscape education of high school students. Digital Landscape Architecture, 336-343.

Selmbacherova, T., Sisler, V., \& Brom, C. (2014). The impact of visual realism on the authenticity of educational simulation: A Comparative Study. Proceedings of the 8th European Conference on Games Based Learning, 520-528. 
Smeureanu, I., \& Isăilă, N. (2017). Innovative educational scenarios in game-based teaching and learning. Amfiteatru Economic, 19 (46), 890-899.

Solarski, C. (2013). Aesthetics of game art and game design. Retrived on 20 May 2017. Soylucicek, S. (2012). Graphic design on educational computer games. Procedia - Social and Behavioral Sciences, 46, 2083-2087.

Whitton, P. (2011). The impact of visual design quality on game-based learning. In Khine, M. (Eds.). Playful Teaching, Learning Games: New Tool for Digital Classrooms. pp. 1-20. Rotterdam, Netherlands: Sense Publishers. 